Check for updates

Cite this: DOI: 10.1039/d1tb00802a

\title{
Modulation of inflammation by anti-TNF $\alpha$ mAb-dendrimer nanoparticles loaded in tyramine-modified gellan gum hydrogels in a cartilage-on-a-chip model
}

\author{
I. M. Oliveira, ${ }^{a b}$ M. R. Carvalho, ${ }^{a b}$ D. C. Fernandes, ${ }^{a b}$ C. M. Abreu, (D) ab \\ F. R. Maia, (D) ${ }^{a b}$ H. Pereira, ${ }^{\text {abcd }}$ D. Caballero, (D) ${ }^{a b}$ S. C. Kundu, ${ }^{a b}$ R. L. Reis ${ }^{a b}$ and \\ J. M. Oliveira (iD *ab
}

\begin{abstract}
Rheumatoid arthritis (RA) is an autoimmune and chronic inflammatory disease characterized by joint inflammation. Since the inflammatory condition plays an important role in the disease process, it is important to develop and test new therapeutic approaches that specifically target and treat joint inflammation. In this study, a human 3D inflammatory cartilage-on-a-chip model was established to test the therapeutic efficacy of anti-TNF $\alpha$ mAb-CS/PAMAM dendrimer NPs loaded-Tyramine-Gellan Gum in the treatment of inflammation. The results showed that the proposed therapeutic approach applied to the human monocyte cell line (THP-1) and human chondrogenic primary cells (hCH) cell-based inflammation system revealed an anti-inflammatory capacity that increased over 14 days. It was also possible to observe that Coll type II was highly expressed by inflamed $\mathrm{hCH}$ upon the culture with antiTNF $\alpha$ mAb-CS/PAMAM dendrimer NPs, indicating that the $\mathrm{hCH}$ cells were able maintain their biological function. The developed preclinical model allowed us to provide more robust data on the potential therapeutic effect of anti-TNF $\alpha$ mAb-CS/PAMAM dendrimer NPs loaded-Ty-GG hydrogel in a physiologically relevant model.
\end{abstract}

Received 10th April 2021

Accepted 7th May 2021

DOI: 10.1039/d1tb00802a

rsc.li/materials-b of RA, many patients do not reach continued clinical remission or become resistant to drug therapy. ${ }^{6,7}$

In vivo animal models are considered the gold standard in preclinical studies of pathophysiological mechanisms of RA. ${ }^{8,9}$ Although animal models present many aspects of human arthritic diseases and are highly useful for testing new therapeutic approaches, they show some limitations, such as the development of arthritis only in predisposed strains of rodents. ${ }^{10,11}$ They present limited development of arthritis, and pathophysiology in animals does not fully mimic the human pathogenic disease. ${ }^{12,13}$ Furthermore, an increased amount of evidence suggest that current animal models are inadequate for wide drug screening due to their low reproducibility in clinical trials due to interspecies variations. ${ }^{14,15}$ Thus, better models are crucial to help improve our knowledge on the pathological mechanisms of RA at pre-clinical level, as well as to develop and test new therapeutic approaches in order to meet patient and medical needs. ${ }^{16,17}$ Having these considerations in mind, research has evolved into the next-generation in vitro screening platform based on the development of microphysiologically relevant systems, such as tissue- and organs-on-chips. ${ }^{18}$ That models are generally based on primary or patient-derived cells, and can better mimic the disease and its treatment, 
therefore promoting the translational process to humans, while decreasing the number of animals experiments. ${ }^{19,20}$ The purpose of such type of in vitro technology is to provide an artificial testing system that fully mimics an tissue, organ, or the human body. ${ }^{21}$ The chip platform can allow regulating indirect and direct cell-cell communication as well as biomechanical signals using several strategies such as the generation of a gradient flow. $^{22}$ The use of microfluidic-based technology thus can address the handicaps between in vitro and in vivo models presenting new promising approaches to research in medicine. ${ }^{23,24}$

In our previous work, monoclonal anti-TNF $\alpha$ antibody (anti-TNF $\alpha \mathrm{mAb}$ ) linked to chondroitin sulfate (CS) modified poly(amidoamine) (CS/PAMAM) dendrimer nanoparticles (NPs) ${ }^{25}$ and loaded into Tyramine-Gellan Gum (Ty-GG) hydrogels were developed as a promising drug delivery vehicle to improve therapeutic efficacy in the treatment of inflammatory conditions such as RA. The therapeutic efficacy was evaluated using an inflammation in vitro model under standard static conditions and dynamic conditions using a bioreactor. In static and dynamic conditions, the Ty-GG hydrogel encapsulated with anti-TNF $\alpha$ mAb-CS/PAMAM dendrimer NPs exhibited good anti-inflammatory activity over time, motivating us to study the efficacy of this approach in the treatment of inflammation in a more physiologically relevant in vitro system, such as a $3 \mathrm{D}$ microfluidic platform. In this context, a new in vitro human $3 \mathrm{D}$ inflammatory cartilage-on-a-chip model was developed aiming to be used as a drug screening platform. An inflammatory environment was established by means of culturing human primary chondrocytes exposed to active pro-inflammatory macrophages, and the anti-TNF $\alpha$ mAb-CS/PAMAM dendrimer NPs loaded-Ty-GG hydrogel was used to test its antiinflammatory therapeutic efficacy.

Of the best of our knowledge, this is the first cartilage-onchip model using the combination of several fields such as nanotechnology, microfluidics and natural based biomaterials.

This work pretends to evolve into the next-generation of in vitro screening platforms in order to meet patient and medical needs.

\section{Material and methods}

\section{In vitro studies under a 3D microfluidic platform}

To mimic the human cartilage inflammation microenvironment, a complex 3D microfluidic chip-based in vitro model was developed. In this sense, human monocyte cell line (THP-1) and human chondrogenic primary cells $(\mathrm{hCH})$ were used in this study, by means of encapsulation in Matrige ${ }^{\circledR}$ and further perfuse in the microfluidic platform.

\section{Chondrogenic cell isolation}

The hCH cells (passage $=3$ ) previously isolated and characterized (kindly provided by Raphael Canadas) were used for these assays. $^{26}$ Briefly, they were obtained from human cartilage during arthroscopic surgeries on male and female donors with ages between 19 and 56 years (Centro Hospitalar Póvoa do
Varzim). The articular cartilage was removed from the bone and cut into small pieces. The samples were washed several times with $1 \%$ PBS/antibiotic-antimycotic (v/v) solution, digested with $0.08 \%$ collagenase type II (Sigma-Aldrich, USA)/ DMEM-F12 medium 1:1 (v/v) and incubated at $37{ }^{\circ} \mathrm{C}$ in a water bath overnight with gentle agitation. The digested tissue was filtered, and cell suspension centrifuged at $500 \mathrm{G}$ for 5 minutes. The isolated cells were then plated in flasks cultured in DMEM-F12 medium and kept in the $\mathrm{CO}_{2}$ incubator at $37{ }^{\circ} \mathrm{C}$ until they reach the desired confluence.

\section{Cell culture}

hCH cells were expanded in DMEM/F12 medium (Dulbecco's Modified Eagle Medium: Nutrient Mixture F-12, Alfagene, Portugal), supplemented with $10 \%$ of Heat Inactivated Fetal Bovine Serum (FBS) (Alfagene, Portugal), sodium bicarbonate (Sigma-Aldrich, USA) and $1 \%(\mathrm{v} / \mathrm{v})$ antibiotic-antimycotic (Alfagene, Portugal), under standard culture conditions (at $37{ }^{\circ} \mathrm{C}$ in a $5 \% \mathrm{CO}_{2}$ incubator).

THP-1 cells (SIGMA, USA) were expanded in RPMI 1640 Medium, GlutaMAX ${ }^{\mathrm{TM}}$ Supplement, HEPES (Thermo Fisher Scientific, USA), supplemented with $10 \%$ of FBS and $1 \%$ of antibiotic-antimycotic, under standard culture conditions $\left(37{ }^{\circ} \mathrm{C}\right.$ in a humidified atmosphere containing $\left.5 \% \mathrm{CO}_{2}\right)$.

\section{Production and encapsulation of anti-TNF $\alpha$ mAb-CS/PAMAM dendrimer NPs into Ty-GG hydrogel}

Anti-TNF $\alpha$ mAb-CS/PAMAM dendrimer NPs and Ty-GG were produced as previously described. ${ }^{27,28}$ Herein, these systems were used to obtain anti-TNF $\alpha$ mAb-CS/PAMAM dendrimer NPs loaded-Ty-GG hydrogel. The hydrogel was prepared by mixing $1 \%(w / v)$ of Ty-GG solution with anti-TNF $\alpha$ mAb-CS/PAMAM dendrimer NPs at a final concentration of $0.5 \mathrm{mg} \mathrm{mL}^{-1}$. Then, the enzymatic crosslinking was made by adding horseradish peroxidase (HRP) solution $\left(0.84 \mathrm{mg} \mathrm{mL}^{-1}\right)$ (Sigma-Aldrich, USA) and hydrogen peroxide solution $\left(\mathrm{H}_{2} \mathrm{O}_{2}\right)(0.36 \%(\mathrm{v} / \mathrm{v}))$ (VWR, USA) prepared in PBS and water, respectively. mAb-CS/PAMAM dendrimer NPs loaded-Ty-GG hydrogel (hereafter designated NPs-Hydrogel) was used in the subsequent assays.

\section{Seeding on microfluidic chip}

To perform cell seeding, the microfluidic platform 3D Cell Culture Chips DAX-1 (Aim Biotech, Singapore) was used. The device consists of three compartments: two lateral channels, where THP-1 (left side) and hCH cells (right side) encapsulated in Matrigel mimic the cartilage inflammation microenvironment, and one central channel where the produced NPs-Hydrogel was injected to treat the inflammatory environment. The appropriate culture medium was placed in the lateral channels with a volume differential ( $90 \mu \mathrm{L}$ at inlet and $70 \mu \mathrm{L}$ at the outlet) to allow fluid diffusion between the channels.

\section{Encapsulation of gelatin in central channel}

Gelatin from porcine skin (Sigma-Aldrich, USA) at $2 \%(\mathrm{w} / \mathrm{v})$ was dissolved in water at $50{ }^{\circ} \mathrm{C}$ and added to the central channel of the microfluidic device. This polymer was used as sacrificial 
hydrogel, preventing the Matrigel ${ }^{\circledR}$ with encapsulated cells to leak into the central chamber. The device was placed at $4{ }^{\circ} \mathrm{C}$ for 20 minutes for gelification.

\section{Validation of the co-culture by fluorescence imaging}

Before the seeding in the microfluidic device, THP-1 and hCH cells were made fluorescent by means of CellTracker CM-Dil Dye (Alfagene, Portugal) and CellTracker Green CMFDA Dye, respectively. hCH confluent cells were detached from the cell culture flasks using trypsin $(0.25 \% \quad(\mathrm{v} / \mathrm{v})$ trypsin-EDTA solution), centrifuged at $300 \mathrm{G}$ for 5 minutes, and diluted in a new cell suspension. THP-1 non-adherent cells were also centrifuged at $300 \mathrm{G}$ for 5 minutes and diluted in $1 \mathrm{~mL}$ of PBS. After this, the THP- 1 and hCH cells were used at a density of $2 \times 10^{6}$ cells $\mathrm{mL}^{-1}$. THP- 1 were incubated with CellTracker CM-Dil Dye $(2 \mu \mathrm{M})$ (Alfagene, Portugal) and hCH with CellTracker Green CMFDA Dye $(1 \mu \mathrm{M})$ (Alfagene, Portugal), for 30 minutes at $37^{\circ} \mathrm{C}$. Cells were then centrifuged, encapsulated in Matrigel ${ }^{\circledR}$ and placed in the lateral channels of the chip. RPMI medium with $100 \mathrm{nM}$ phorbol 12-myristate-13-acetate (PMA) (Sigma-Aldrich, USA) was added to the channel with THP-1 cells, to differentiate them into macrophage phenotype, and DMEM-F12 was added to the channel with hCH cells. The microfluidic device was incubated for 72 hours and observed under fluorescence microscopy (Dil tracker ex/em 553/570 nm Green tracker ex/em 492/517 nm) in the Fluorescence Inverted Microscope (Axio Observer, Zeiss). Images were acquired using the Zen microscope processing software, connected to the digital camera Axio Observer.

\section{Encapsulation of hCH and THP-1 in lateral channels to stimulate an inflammatory environment}

The hCH and THP-1 cells were centrifuged at $300 \mathrm{G}$ for 5 minutes and diluted in a new cell suspension with appropriate media. Then, the THP-1 and hCH cells were encapsulated in Matrige ${ }^{\mathbb{R}}$ at a density of $2 \times 10^{6}$ cells $\mathrm{mL}^{-1}$. THP-1 cells suspended in Matrigel ${ }^{\mathbb{B}}$ were injected in the left channel and hCH in the right channel of the microfluidic device, followed by incubation for 20 minutes at $37{ }^{\circ} \mathrm{C}$ for crosslinking. After this, RPMI medium with 100 nM PMA was added to the channel with THP-1 cells and DMEM-F12 was added to the channel with hCH cells to prevent drying. A differential of cell culture medium was added to the inlet $(90 \mu \mathrm{L})$ and outlet $(70 \mu \mathrm{L})$ of the microchannel to allow perfusion and nutrition of the cells. The microfluidic devices were incubated for 24 hours, after which the medium was replaced with RPMI medium without PMA in the THP-1 channel and DMEM-F12 in hCH channel, followed by another 24 hours of incubation.

To enable an inflammatory response, THP-1 cells were incubated for 5 hours with $100 \mathrm{ng} \mathrm{mL} \mathrm{m}^{-1}$ of lipopolysaccharide (LPS) (SigmaAldrich, USA) in RPMI medium. After incubation time, gelatin was flushed from the central channel with PBS at $50{ }^{\circ} \mathrm{C}$, letting the channel free to add our formulation of NPs-Hydrogel, for 3 and 7 days. In parallel, a positive control chip comprising THP-1 stimulated with LPS in left channel, and $\mathrm{hCH}$ in the right channel without NPs-Hydrogel was used (hereafter designated $\mathrm{CTRL}^{+}$). Additionally, a negative control chip with THP-1 differentiated with PMA in the left channel, and hCH cells in the right channel without NPs-hydrogel was used (hereafter designated $\mathrm{CTRL}^{-}$).

\section{Alamar blue assay}

Alamar blue was performed to assess the effect of NPs-Hydrogel in terms of metabolic activity in THP- 1 and hCH cells for 3 and 7 days. For the Alamar blue metabolic activity assay in 3D, cells encapsulated in Matrigel ${ }^{\circledR}$ were retrieved using Corning Cell Recovery Solution (Laborspirit, Portugal) for 40 minutes at $4{ }^{\circ} \mathrm{C}$. After complete Matrigel ${ }^{\circledR}$ release, cells were collected to an Eppendorf tube and centrifuged to a pellet ( $300 \mathrm{G}$ for 5 minutes). The supernatant was removed and a culture medium containing $10 \%$ (v/v) of AlamarBlue ${ }^{\circledR}$ (BioRad, Oxford, UK) was added. The tubes were kept in the dark, at $37{ }^{\circ} \mathrm{C}$ in the $\mathrm{CO}_{2}$ incubator for 4 hours. Afterwards, $100 \mu \mathrm{L}$ of each condition were transferred to 96-well plates. The fluorescence was read at an excitation wavelength of 530/25 $\mathrm{nm}$ and at an emission wavelength of 590/535 nm, using a microplate reader (Synergy HT, BioTek, Instruments, USA).

\section{DNA quantification}

The proliferation of THP-1 and hCH cells in contact with NPs-Hydrogel for 3 and 7 days were analyzed by means of DNA quantification. For DNA quantification, cells encapsulated in Matrigel ${ }^{\circledR}$ were retrieved using Corning Cell Recovery Solution for 40 minutes at $4{ }^{\circ} \mathrm{C}$ and the subsequent protocol was performed as previously described. After centrifugation, the supernatant was removed, and ultrapure water was added. The cells' lysate solution was stored at $-80{ }^{\circ} \mathrm{C}$ until further analysis. Quanti-IT PicoGreen dsDNA Assay Kit (Alfagene, Portugal) was used to quantify dsDNA, according to manufacturers' instructions. Then, the plate was incubated in the dark for 10 minutes and the fluorescence was read using excitation of $480 / 20 \mathrm{~nm}$ and emission of 528/20 nm, in a microplate reader (SYNERGY HT, BIO-TEK). DNA concentration was determined using a standard curve in the range of 2 to $0 \mu \mathrm{L} \mathrm{mL} L^{-1}$.

\section{Live/dead staining}

Cell viability of THP-1 and hCH cells cultured in the microfluidic chip's lateral channels was assessed in the presence of NPs-Hydrogel for 3 and 7 days, using the live/dead assay. Initially, the NPs-Hydrogel was flushed with PBS at $50{ }^{\circ} \mathrm{C}$ in the central channel to add the solution of Calcein-AM (1 mM; live cells in green) (Alfagene, Portugal) and ethidium homodimer (EthD-1 6 mM; dead cells in red) (Laborspirit, Portugal). The chips were incubated for 40 minutes in the dark, at $37{ }^{\circ} \mathrm{C}$ in the $\mathrm{CO}_{2}$ incubator, to reach the cells in the lateral channels. After this, the central channel was washed with PBS three times. The chips were observed under fluorescence microscopy (EthD-1 ex/em 528/617 nm Calcein-AM; ex/em 495/515 nm) in the Fluorescence Inverted Microscope. Images were acquired using the Zen microscope processing software, connected to the digital camera Axio Observer. A Z-stack function was used to combine images at different depths into one final image. 
Anti-inflammatory activity of anti-TNF $\alpha$ mAb-CS/PAMAM dendrimer NPs loaded-Ty-GG hydrogel

To assesses the therapeutic efficacy of NPs-Hydrogel, at each time point ( 3 and 7 days), the culture medium was collected and stored at $-80{ }^{\circ} \mathrm{C}$ until further analysis. Human TNF-alpha DuoSET ELISA (R\&D Systems, USA) kit and DuoSet Ancillary Reagent Kit 2 (R\&D Systems, USA), for the optimum performance of the ELISA kit were used to evaluate the anti-inflammatory activity. TNF $\alpha$ standard solutions with concentrations from

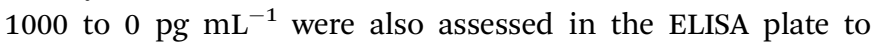
perform the calibration curve. The optical density at $450 \mathrm{~nm}$ was read in a microplate reader (Synergy HT, BIO-TEK, Winooski, VT, USA).

\section{Immunofluorescence staining}

To evaluate the amount of Collagen Type II (Coll type II) present in hCH primary cells, immunofluorescence staining was performed. On the 7th day, the NPs-Hydrogel was flushed from the central channel of the chip with PBS at $50{ }^{\circ} \mathrm{C}$. For cell fixation, $10 \%$ formaldehyde was added and left to incubate for 15 minutes at RT. Then, the channel was washed twice with PBS. For permeabilization $0.1 \%$ Triton X-100 was added and incubated for 10 minutes at RT. Then, the channel was washed twice with PBS. For blocking, the PBS was removed and replaced with blocking buffer (2\% BSA in PBS) and left to incubate for 2 hours at RT. The blocking buffer was removed from the central channel and mouse anti-human COL II monoclonal antibody (Laborspirit, Portugal) in PBS solution $(1: 250)$ was added and incubated overnight at $4{ }^{\circ} \mathrm{C}$. Then, the central channel was washed 3 times with PBS and the secondary antibody Alexa Fluor ${ }^{\circledR} 594$ donkey anti-mouse (1:500) (Invitrogen, USA) with DAPI $(1: 1000)$ (VWR International, USA), in PBS solution and was left to incubate 1.5 hours. The samples were immediately analyzed by confocal microscopy (Leica, SP8, Germany) (Alexa Fluor 594: ex/em 590/617 nm; DAPI: ex/em 358/461 nm).

\section{Statistical analysis}

Statistical analysis was performed with GraphPad Prism 8 version, where a Shapiro-Wilk normality test was done to assess the data normality. Non-parametric Kruskal-Wallis test was applied to all assays. Statistical significance was obtained as ${ }^{*} p<0.05$. All results are presented as means \pm standard deviations, and all assays were performed in triplicate.

\section{Results and discussion}

\section{Development of a 3D inflammatory cartilage-on-a-chip model}

In this study, an in vitro 3D inflamed cartilage model-on-a-chip to test the therapeutic efficacy of NPs-Hydrogel was established. The first steps consisted in the encapsulation of proinflammatory THP- 1 cells in Matrige ${ }^{\circledR}$ in one of the lateral channels of the chip to mimic the intended inflammatory environment, while $\mathrm{hCH}$ cells encapsulated in Matrigel $^{\mathbb{R}}$ were seeded on the other microchannel, as target cells. NPs-Hydrogel was added to be used as a treatment option.

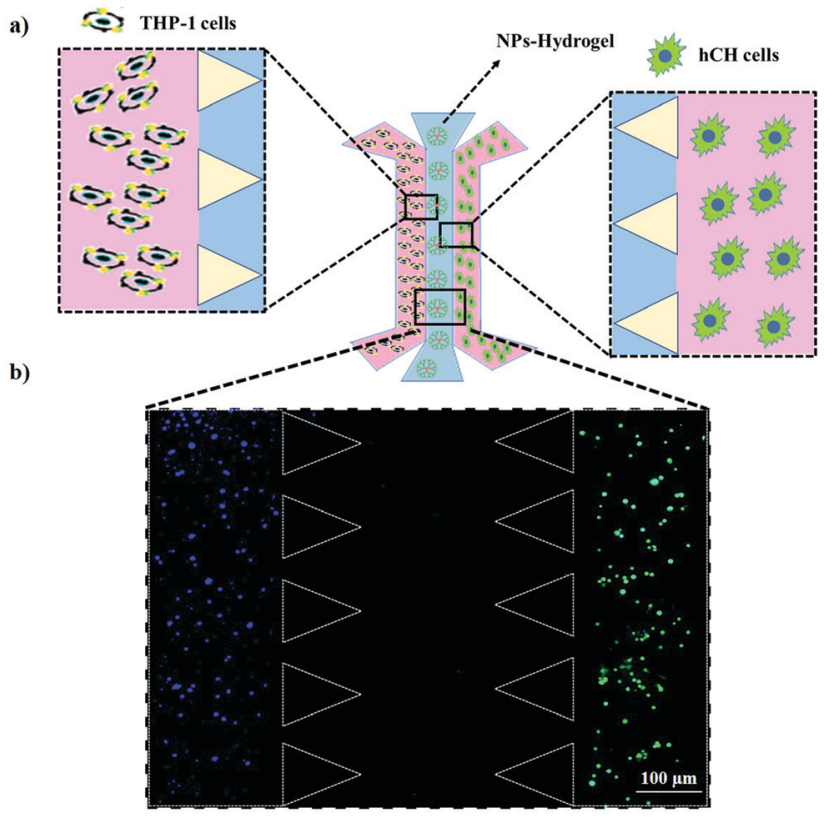

Fig. 1 Design of microfluidic chip. (a) Schematic representation of a 3D inflamed cartilage model-on-a-chip. (b) Fluorescence microscopy image of microfluidic lateral channel mimicking inflamed cartilage comprising THP-1 cells embedded in Matrigel ${ }^{\circledR}$ (left channel, stained with blue) and $\mathrm{hCH}$ cells (right channel, stained with green), after 3 days of culture (scale bar $=100 \mu \mathrm{m})$

In the sense, anti-TNF $\alpha$ mAb-CS/PAMAM dendrimer NPs are released from the hydrogel and the biological effect was evaluated.

In order to achieve this, the traditional "sandwich" microfluidic design was chosen. ${ }^{29}$ The selected microfluidic has a central chamber and two perfusable lateral channels. THP-1 cells encapsulated in Matrigel ${ }^{\circledR}$ were seeded in the left channel, hCH were seeded in the right channel, and NPs-Hydrogel was added in the central channel (Fig. 1a).

To assess the achievement and maintenance of the culture for 3 days, THP- 1 cells encapsulated in Matrige ${ }^{\circledR}$ were labelled with CellTracker CM-Dil Dye (Blue) and hCH with CellTracker Green CMFDA Dye (Green), as shown in (Fig. 1b). It was possible to observe that the cells remained in the lateral channels and were well distributed along the channel in the Matrigel $^{\mathbb{R}}$ after 3 days of culture.

\section{Influence of anti-TNF $\alpha$ mAb-CS/PAMAM dendrimer NPs loaded-Ty-GG hydrogel on metabolic activity and cell proliferation of THP-1 and hCH cells}

Cell viability was assessed by means of Live/Dead assay for 3 and 7 days. At each time point, live (green) and dead (red) THP-1 and hCH cells were stained by adding Calcein/EthD and the chips were observed under fluorescence microscopy (Fig. 2). As observed in Fig. 2a, at day 3, all the conditions tested showed that THP-1 cells differentiated with PMA (healthy cells CTRL ${ }^{-}$), stimulated with LPS (inflamed cells $\mathrm{CTRL}^{+}$) and inflamed THP-1 cells with NPs-Hydrogel remained viable (all green cells) and no dead cells (red) were found. So, it was possible to verify 


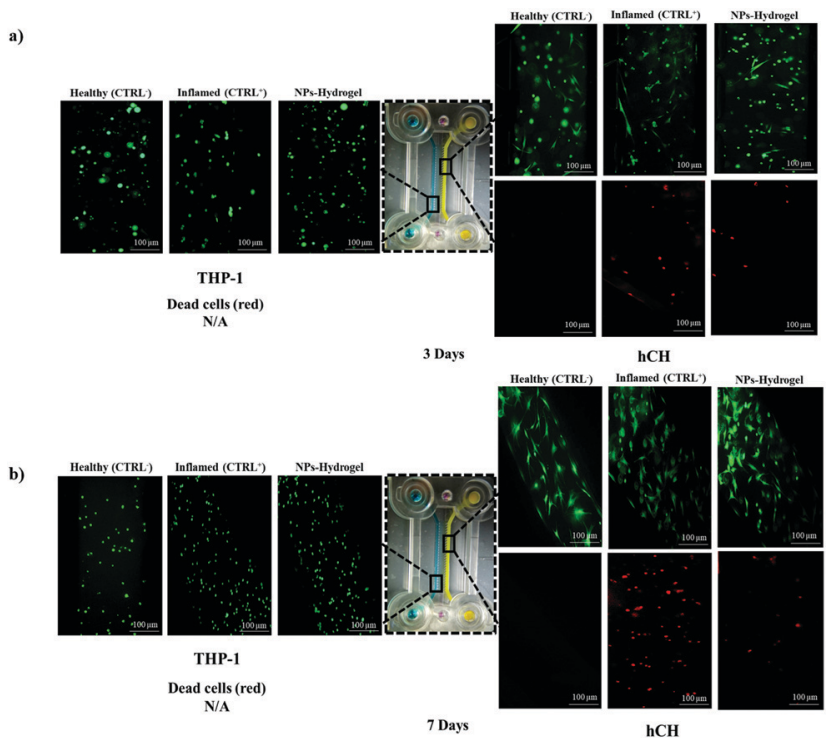

Fig. 2 Live/dead assay of THP-1 and hCH cells. Healthy CTRL ${ }^{-}$(THP-1 differentiated with PMA cultured with $\mathrm{hCH}$ ); Inflamed $\mathrm{CTRL}^{+}$(THP-1 stimulated with LPS cultured with $\mathrm{hCH}$ ); and NPs-Hydrogel (inflamed THP-1 cultured with hCH with NPs-Hydrogel treatment) for 3 (a) and 7 days (b).

that inflamed THP-1 cells remain viable, as seen in other studies $^{30,31}$ and that NPs-Hydrogels have no cytotoxic effects on cells.

Furthermore, the healthy THP-1 cells did not affect the viability of hCH with no cell death detected. However, hCH cells in the presence of inflamed THP-1 cells showed cell death. The same pattern was seen in inflamed THP-1 with NPs-Hydrogel, but the incidence of dead chondrocytes appears to be lower. On the seventh day (Fig. 2b), all the THP-1 conditions maintained a high incidence of cell viability. In addition, it was possible to observe a higher chondrocytes adhesion through the observation of the cytoplasmic membrane and a higher incidence of dead chondrocytes was verified in the inflamed THP-1 condition. As expected, the healthy THP-1 cells did not affect the viability of hCH cells over time. Furthermore, inflamed THP-1 cells subjected to the presence of NPs-Hydrogel also did not affect the cell viability. Moreover, in an inflammatory environment, it allowed to reduce the cell death of chondrocytes, showing the possible therapeutic efficacy of the approach under study. However, inflamed THP-1 cells induced hCH cell death. Thus, these results are in agreement with other studies. ${ }^{32,33}$ In fact, inflammatory environment induces chondrocytes cell death, as seen in patients with RA. ${ }^{32,33}$

Cells' metabolic activity and proliferation were determined by means of performing Alamar blue and DNA quantification assays, respectively, for 3 and 7 days (Fig. 3). As depicted in Fig. 3a, at the first time point, THP-1 cells (differentiated THP-1, inflamed THP-1, and inflamed THP-1 cells with NPs-Hydrogel) showed high metabolic activity with no significant differences between conditions. In addition (Fig. $3 \mathrm{~b}$ ), it was possible to verify that the healthy THP-1 cells did not negatively affect the metabolic activity of chondrocytes. However, inflamed THP-1 cells

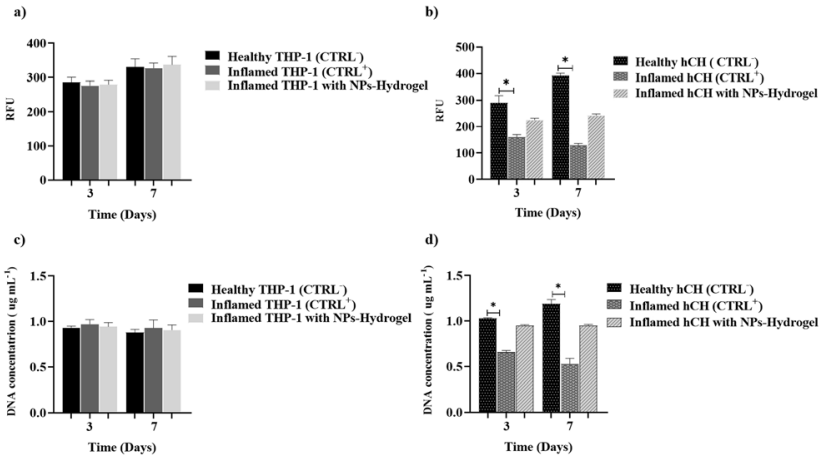

Fig. 3 Evaluation of metabolic activity and proliferation of THP-1 and hCH cells. (a) Metabolic activity assessed within the three conditions: Healthy THP-1 (CTRL $\left.{ }^{-}\right)$cultured with hCH, Inflamed THP-1 (CTRL $\left.{ }^{+}\right)$cultured with $\mathrm{hCH}$, and Inflamed THP-1 cultured with $\mathrm{hCH}$, and with NPs-Hydrogel treatment for 3 and 7 days. (b) Cell proliferation assessed by DNA quantification within the three conditions: Healthy THP-1 (CTRL $\left.{ }^{-}\right)$cultured with $\mathrm{hCH}$, Inflamed THP-1 $\left(\mathrm{CTRL}^{+}\right)$cultured with $\mathrm{hCH}$, and Inflamed THP-1 cultured with $\mathrm{hCH}$, and with NPs-Hydrogel treatment for 3 and 7 days. Data shown as Mean \pm SD. * denotes statistical significant difference $p<0.05$.

negatively and significantly affected the metabolic activity of chondrocytes as compared to the previous condition, as expected. $^{32,33}$ In inflamed THP-1 cells with NPs-Hydrogel, despite a slight decrease, there was no significant difference in metabolic activity. On the seventh day of culture, there was a slight increase in metabolic activity in all conditions with THP-1 cells, with no significant differences between them. As expected, there was an increase in the metabolic activity in the chondrocytes when were in contact with healthy THP-1 cells and inflamed THP-1 cells with NPs-Hydrogel. Nevertheless, inflamed THP-1 cells induced a significant decrease in the metabolic activity of chondrocytes, when compared with healthy THP-1 cells, corroborating previous Live/Dead results (Fig. 2a and b).

DNA quantification was also analyzed to assess THP-1 and hCH cells' proliferation in contact in the different conditions for 3 and 7 days. As depicted in Fig. 3c, none of the conditions negatively affected the proliferation of THP-1 cells after $3 \mathrm{~d}$ of culture. However, it was observed a decrease in the proliferation of THP- 1 cells in all conditions, after 7 days of culture. These results are in agreement with other studies ${ }^{34,35}$ wherein PMA induces differentiation of cells and while cells are spending energy to differentiate. Furthermore (Fig. 3d), it was possible to verify that healthy THP-1 cells also did not affect the chondrocytes metabolic activity, observing an increase in proliferation over time. The hCH cells in contact with inflamed THP-1 cells with NPs-Hydrogel maintain a constant profile of proliferation over time. In addition, inflamed THP-1 cells significantly decreased cell proliferation of chondrocytes as compared to healthy cells over time, corroborating the results obtained from Alamar blue observations.

\section{Anti-TNF $\alpha$ mAb-CS/PAMAM dendrimer NPs loaded-Ty-GG hydrogel to modulate the inflammatory environment}

In our previous work, NPs-Hydrogel was developed. This system demonstrated a good anti-inflammatory activity over time, 
allowing the retention of a high percentage of $\mathrm{TNF} \alpha$ present within the inflammatory environment. For more reliable testing of the effectiveness of this approach in a more physiologically-relevant microenvironment, the microfluidic device was used as a preclinical model for drug screening.

In order to assess the therapeutic efficacy of NPs-Hydrogel in inflamed THP-1 and hCH cells, the levels of free TNF $\alpha$ in the medium of 7 days of culture were quantified using ELISA KIT (Fig. 4). THP-1 cells differentiated with PMA (healthy cells) were used as the negative control, and THP-1 stimulated with LPS were used as the positive control (inflamed cells). On day 3, the results demonstrated that the induction of inflammation was successful, with a low TNF $\alpha$ amount present in healthy THP-1 cells and high TNF $\alpha$ amount in inflamed THP-1 cells, with significant differences between the conditions. Furthermore, in the inflamed THP-1 cells with NPs-Hydrogel condition, there was a decrease in the free TNF $\alpha$ in the medium when compared with inflamed cells. However, there was no significant differences as compared with healthy cells. hCH cells in a healthy THP-1 environment also showed low amounts of TNF $\alpha$ present in the medium but in contrast, hCH in an inflamed THP-1 environment demonstrated high levels of free $\mathrm{TNF} \alpha$, with significant differences between the conditions. This means that the mimicry of the inflammatory environment in the cartilage was successful. In the inflamed THP-1 environment with NPs-Hydrogel, a low amount of $\mathrm{TNF} \alpha$ in hCH cells was found in the culture medium when compared to the inflamed and untreated environment. On day 7, in the condition with inflamed THP-1 cells and NPs-Hydrogel, the amount of free TNF $\alpha$ present in the medium decreased significantly when compared to the inflamed THP-1 condition without treatment. Furthermore, the same behavior was found in chondrogenic cells with NPs-Hydrogel, where the amount of free $\mathrm{TNF} \alpha$ in the medium decreased substantially when compared to the inflamed hCH condition. So, these results show that the inflamed THP-1 cells induced the production of an inflammatory environment in chondrocytes, indicating that the production of the inflammatory cartilage model was successfully achieved. In addition, this preclinical model allowed to reveal the possible therapeutic effect of NPs-Hydrogel in terms of protecting the chondrocytes from inflammation, corroborating the results obtained in other studies. ${ }^{36,37}$ In fact, anti-tumor necrosis factor

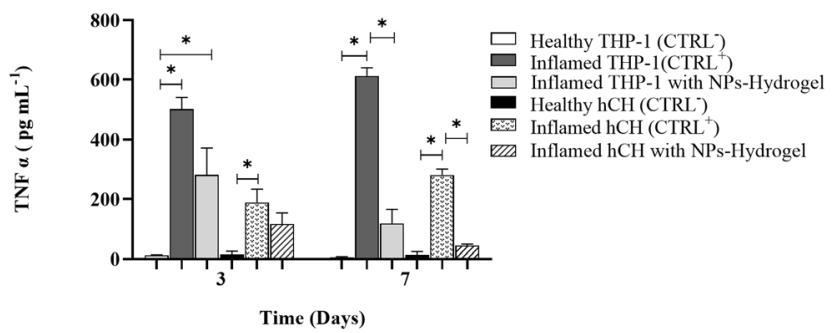

Fig. 4 Quantification of free TNF $\alpha$ in the culture medium. Amount of free TNF $\alpha$ within the three conditions: healthy THP-1 (CTRL $\left.{ }^{-}\right)$cultured with hCH; Inflamed THP-1 $\left(\mathrm{CTRL}^{+}\right)$cultured with $\mathrm{hCH}$; and Inflamed THP-1 cultured with $\mathrm{hCH}$, and with NPs-Hydrogel treatment for 3 and 7 days. Data shown as Mean $\pm S D$. * denotes statistical significant difference $p<0.05$.
(TNF) therapies have been successfully used in the treatment of RA. ${ }^{36,37}$ Importantly, it was shown that even linked to dendrimer NPs and encapsulated in the hydrogel, they maintain therapeutic efficacy over time.

\section{Collagen type II as a marker of chondrocyte functionality}

Articular cartilage is composed of chondrocytes surrounded by a specialized matrix constituted predominantly of Coll type II and proteoglycan. ${ }^{38,39}$ Rheumatoid Arthritis is a chronic inflammatory disease characterized by joint inflammation that induces cartilage destruction. ${ }^{40,41}$ As Coll type II degradation may indicate cartilage destruction in the joints of RA patients, it is an important parameter to evaluate since it can be useful as a disease-specific biomarker. ${ }^{42,43}$

In this sense, the immunofluorescence staining was performed to qualitatively evaluate the presence of Coll type II in the chondrogenic cells matrix, under the three conditions: healthy THP-1 cells cultured with hCH, inflamed THP-1 cultured with $\mathrm{hCH}$, and inflamed THP-1 cultured with hCH, and with NPsHydrogel treatment after 7 days of culture (Fig. 5). The results demonstrated that the state of THP-1 cells can influence the Coll type II marker in the hCH, being highly expressed in conditions where hCH were in contact with healthy THP-1 cells and with inflamed THP-1 cells with NPs-Hydrogel. However, in an inflammatory environment without NPs-Hydrogel, the incidence of Coll type II was very low, being in agreement with what was previously mentioned: the inflammation process induces Coll type II degradation. ${ }^{42,43}$ These results can suggest that the possible therapeutic efficacy of NPs-Hydrogel in this approach.

Despite the promising results, these assays were performed in normoxia conditions. However, hypoxia is the predominant microenvironment characteristic in inflamed cartilage. ${ }^{44,45}$ The increased oxygen consumption by inflamed cells and the infiltration of the immune cells with an interrupted blood supply caused by vascular dysfunction leads to tissue hypoxia in RA. ${ }^{46,47}$ In this sense would be interesting, and will be the focus of future works, to evaluate this preclinical model in a hypoxic
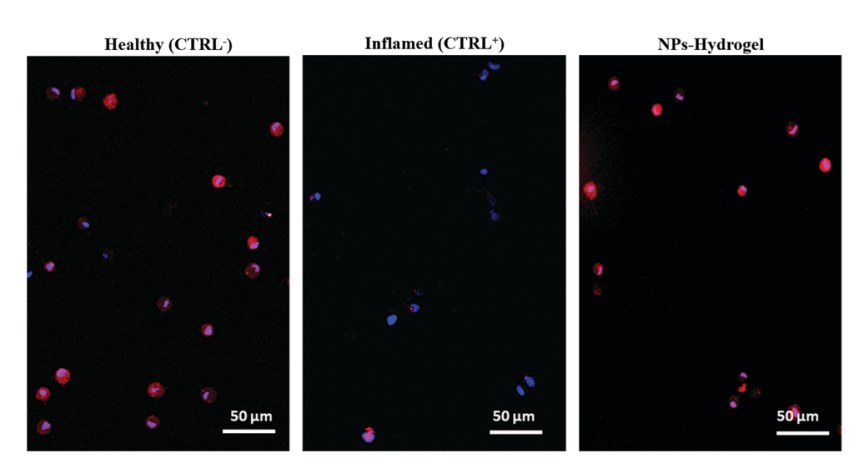

Fig. 5 Immunofluorescence staining of Coll type II in the chondrogenic cells matrix. Coll type II expression was assessed in $\mathrm{hCH}$ cultured within the three conditions: Healthy $\left(\mathrm{CTRL}^{-}\right)$that corresponds to healthy THP-1 cultured with $\mathrm{hCH}$, Inflamed (CTRL ${ }^{+}$) that corresponds to inflamed THP-1 cultured with $\mathrm{hCH}$, and NPs-Hydrogel that corresponds to inflamed THP-1 cultured with $\mathrm{hCH}$, and with NPs-Hydrogel treatment for 7 days. Coll type II stained in red; Nucleus stained in blue. Scale bar $=50 \mu \mathrm{m}$. 
scenario assess whether it would influence the results, and to mimic more precisely the cartilage inflammation environment.

\section{Conclusion}

Of the best of our knowledge, and up to this date, this was the first time a cartilage-on-chip was developed using at the same time several fields such as nanotechnology, microfluidics and natural based biomaterials in this combination. The relative complexity of this co-culture model can represent both the healthy and the disease scenario in the cartilage during an inflammation case, such as rheumatoid arthritis, depending on whether the THP-1 cells are stimulated to produce an inflammatory environment or not. This was achieved by stimulation of the pro-inflammatory phenotype of THP-1 to mimic the intended inflammatory environment and the use of human chondrogenic cell as the target cells. The NPs-loaded hydrogels were used as anti-inflammatory treatment.

In this work, the results showed that THP-1 and hCH cells encapsulation in Matrigel ${ }^{\circledR}$ and the establishment of the inflammatory environment by co-culturing them on-chip was successful. The THP-1 and hCH cells cultured with NPs-Hydrogel were metabolic active and proliferative along the study time. Furthermore, Live/Dead assays corroborated these results and showed that cell death was lower in hCH cell-based inflammation system cultured with NPs-Hydrogel when compared to the inflamed environment without NPs-hydrogel treatment. In addition, the THP-1 and hCH cellbased inflammation model cultured with the therapeutic approach presented an increased anti-inflammatory capacity over time.

It was also possible to observe that Coll type II is highly expressed in hCH cell-based inflammation system cultured with NPs-Hydrogel, while its expression was not observed in a pro-inflammatory environment, indicating that in addition to modulating the inflammatory environment, the hCH maintain biological functionally. These important data supported the results obtained in the previous works on the effectiveness of this system regarding inflammation treatment. So, the preclinical model developed allows us to obtain more robust data on the potential therapeutic of NPs-Hydrogel in an in vivo-like inflammation environment, namely, to be used in the treatment of RA.

Altogether, this work provided valuable information on the delivery of hydrogel-based drug carriers to better understand their clinical potentials prior to the resource intensive animal and clinical studies.

With this manuscript, we have evolved into the nextgeneration of in vitro screening platforms, approaches in order to meet patient and medical needs.

\section{Conflicts of interest}

There are no conflicts to declare.

\section{Acknowledgements}

The authors thank the financial support under the Norte2020 project (NORTE-08-5369-FSE000044). M. R. C. acknowledges TERM RES Hub Ref. Norte-01-0145-FEDER-02219015 working contract. D. C. F. acknowledges Portuguese Foundation for Science and Technology (FCT) for his phD scholarship (PD/ BD/143081/2018) and F. R. M. for her contract under the Transitional Rule DL 57/2016 (CTTI-57/18-I3BS(5)). C. M. A., D. C. V. and S. C. K. thank the support of FCT (PTDC/BTM-ORG/ 28070/2017). D. C. V acknowledges the CEEC individual contract (CEECIND/00352/2017). S. C. K wishes to record the financial support from EU Framework Programme for Research and Innovation H2020 on FoReCaST under grant agreement no. 668983 and BREAST-IT FCT-Portugal project (PTDC/BTM-ORG/ 28168/2017). The FCT distinction attributed to J. M. O. under the Investigator FCT program (number IF/01285/2015) is also greatly acknowledged.

\section{References}

1 Q. Guo, Y. Wang, D. Xu, J. Nossent, N. J. Pavlos and J. Xu, Bone Res., 2018, 6, 15.

2 Y.-J. Lin, M. Anzaghe and S. Schülke, Cells, 2020, 9, 880.

3 C. A. Murphy, A. K. Garg, J. Silva-Correia, R. L. Reis, J. M. Oliveira and M. N. Collins, Annu. Rev. Biomed. Eng., 2019, 21, 495-521.

4 J. Bullock, S. A. A. Rizvi, A. M. Saleh, S. S. Ahmed, D. P. Do, R. A. Ansari and J. Ahmed, Med. Princ. Pract., 2018, 27, 501-507.

5 L.-D. Quan, G. M. Thiele, J. Tian and D. Wang, Expert Opin. Ther. Pat., 2008, 18, 723-738.

6 R. F. Canadas, T. Ren, A. P. Marques, J. M. Oliveira, R. L. Reis and U. Demirci, Adv. Funct. Mater., 2018, 28, 1804148.

7 D. Liu, N. Yuan, G. Yu, G. Song and Y. Chen, Am. J. Transl. Res., 2017, 9, 3758-3775.

8 E. L. Kuyinu, G. Narayanan, L. S. Nair and C. T. Laurencin, J. Orthop. Surg. Res., 2016, 11, 19.

9 B. J. B. Lewis and D. R. Branch, Pharmacology, 2020, 105, 618-629.

10 P. Hawkins, R. Armstrong, T. Boden, P. Garside, K. Knight, E. Lilley, M. Seed, M. Wilkinson and R. O. Williams, Inflammopharmacology, 2015, 23, 131-150.

11 B. D. Fischer, A. Adeyemo, M. E. O'Leary and A. Bottaro, Arthritis Res. Ther., 2017, 19, 146.

12 K. Schinnerling, C. Rosas, L. Soto, R. Thomas and J. C. Aguillón, Front. Immunol., 2019, 10, 203.

13 C. J. Moran, A. Ramesh, P. A. J. Brama, J. M. O’Byrne, F. J. O'Brien and T. J. Levingstone, J. Exp. Orthop., 2016, 3, 1.

14 S. Festing and R. Wilkinson, EMBO Rep., 2007, 8, 526-530.

15 D. B. Fogel, Contemp. Clin. Trials Commun., 2018, 11, 156-164.

16 A. Damerau and T. Gaber, Int. J. Mol. Sci., 2020, 21, 7916.

17 M. L. Andersen and L. M. F. Winter, An. Acad. Bras. Cienc., 2019, 91. 
18 P. Occhetta, A. Mainardi, E. Votta, Q. Vallmajo-Martin, M. Ehrbar, I. Martin, A. Barbero and M. Rasponi, Nat. Biomed. Eng., 2019, 3, 545-557.

19 Y. A. Jodat, M. G. Kang, K. Kiaee, G. J. Kim, A. F. H. Martinez, A. Rosenkranz, H. Bae and S. R. Shin, Curr. Pharm. Des., 2018, 24, 5471-5486.

20 E. W. Esch, A. Bahinski and D. Huh, Nat. Rev. Drug Discovery, 2015, 14, 248-260.

21 Q. Wu, J. Liu, X. Wang, L. Feng, J. Wu, X. Zhu, W. Wen and X. Gong, BioMedical Engineering OnLine, 2020, 19, 9.

22 M. Rothbauer, G. Höll, C. Eilenberger, S. R. A. Kratz, B. Farooq, P. Schuller, I. Olmos Calvo, R. A. Byrne, B. Meyer, B. Niederreiter, S. Küpcü, F. Sevelda, J. Holinka, O. Hayden, S. F. Tedde, H. P. Kiener and P. Ertl, Lab Chip, 2020, 20, 1461-1471.

23 P. Cui and S. Wang, J. Pharm. Anal., 2019, 9, 238-247.

24 J. Collison, Nat. Rev. Rheumatol., 2019, 15, 511.

25 I. M. Oliveira, C. Gonçalves, E. P. Oliveira, R. SimónVázquez, A. da Silva Morais, Á. González-Fernández, R. L. Reis and J. M. Oliveira, Mater. Sci. Eng., C, 2021, 121, 111845.

26 S. I. Correia, J. Silva-Correia, H. Pereira, R. F. Canadas, A. da Silva Morais, A. M. Frias, R. A. Sousa, C. N. van Dijk, J. Espregueira-Mendes, R. L. Reis and J. M. Oliveira, J. Tissue Eng. Regener. Med., 2017, 11, 1949-1962.

27 I. M. Oliveira, C. Gonçalves, E. P. Oliveira, R. SimónVázquez, A. da Silva Morais, Á. González-Fernández, R. L. Reis and J. M. Oliveira, Mater. Sci. Eng., C, 1920, 111845.

28 I. M. Oliveira, C. Gonçalves, M. E. Shin, S. Lee, R. L. Reis, G. Khang and J. M. Oliveira, Drug Delivery Transl. Res., 2020, $1-13$.

29 H.-F. Wang, Y. Liu, T. Wang, G. Yang, B. Zeng and C.-X. Zhao, ACS Biomater. Sci. Eng., 2020, 6, 5040-5050.

30 J. D. Widdrington, A. Gomez-Duran, A. Pyle, M.-H. RuchaudSparagano, J. Scott, S. V. Baudouin, A. J. Rostron, P. E. Lovat,
P. F. Chinnery and A. J. Simpson, Front. Immunol., 2018, 9, 2217.

31 X. Liu, S. Yin, Y. Chen, Y. Wu, W. Zheng, H. Dong, Y. Bai, Y. Qin, J. Li, S. Feng and P. Zhao, Mol. Med. Rep., 2018, 17, 5484-5491.

32 M. B. Goldring and M. Otero, Curr. Opin. Rheumatol., 2011, 23, 471-478.

33 D. Tateiwa, H. Yoshikawa and T. Kaito, Cells, 2019, 8, 818.

34 A. Spano, S. Barni and L. Sciola, Cell Proliferation, 2013, 46, 328-347.

35 E. Richter, K. Ventz, M. Harms, J. Mostertz and F. Hochgräfe, Front. Cell Dev. Biol., 2016, 4, 21.

36 J. Geiler, M. Buch and M. F. McDermott, Curr. Pharm. Des., 2011, 17, 3141-3154.

37 X. Ma and S. Xu, Biomed. Rep., 2013, 1, 177-184.

38 B. D. Boyan, M. Doroudi, K. Scott and Z. Schwartz, in Vitamin D, ed. D. Feldman, Academic Press, 4th edn, 2018, pp. 405-417, DOI: 10.1016/B978-0-12-809965-0.00024-0.

39 A. J. Sophia Fox, A. Bedi and S. A. Rodeo, Sports Health, 2009, 1, 461-468.

40 Y. Tanaka, Inflammation Regener., 2020, 40, 20.

41 E. Macfarlane, M. J. Seibel and H. Zhou, Bone Res., 2020, 8, 33.

42 R. B. M. Landewé, P. Geusens, D. M. F. M. van der Heijde, M. Boers, S. J. van der Linden and P. Garnero, Ann. Rheum. Dis., 2006, 65, 40-44.

43 A. Poole, M. Kobayashi, T. Yasuda, S. Laverty, F. Mwale, T. Kojima, T. Sakai, C. Wahl, S. El-Maadawy and G. Webb, Ann. Rheum. Dis., 2002, 61, ii78-ii81.

44 X. Guo and G. Chen, Front. Immunol., 2020, 11, 1668.

45 E. P. Cummins, C. E. Keogh, D. Crean and C. T. Taylor, Mol. Aspects Med., 2016, 47-48, 24-34.

46 C. T. Taylor and S. P. Colgan, Nat. Rev. Immunol., 2017, 17, 774-785.

47 T. Bodamyali, C. Stevens, M. Billingham, S. Ohta and D. Blake, Ann. Rheum. Dis., 1998, 57, 703-710. 